\title{
Aspectos clínicos, cirúrgicos e epidemiológicos da luxação de patela em cães atendidos no Hospital Veterinário, no período de janeiro de 2000 a julho de 2010: estudo retrospectivo
}

\author{
[Clinical, surgical and epidemiological aspects of the patella luxation in dogs examined at the \\ Veterinary Hospital in the period from January 2000 to July 2010: A retrospective study] \\ J.S. Lara ${ }^{1}$, H.P. Oliveira ${ }^{2}$, E.G.L. Alves ${ }^{1}$, R.F. Silva ${ }^{1}$, C.M.F. Resende ${ }^{2}$ \\ ${ }^{1}$ Aluno de pós-graduação - Escola de Veterinária - Universidade Federal de Minas Gerais - Belo Horizonte, MG \\ ${ }^{2}$ Escola de Veterinária - Universidade Federal de Minas Gerais - Belo Horizonte, MG
}

\begin{abstract}
RESUMO
Avaliaram-se, por meio de estudo retrospectivo, o perfil epidemiológico, os sinais clínicos, as técnicas cirúrgicas utilizadas e a recuperação de cães apresentados para tratamento de luxação de patela no Hospital Veterinário da UFMG, no período de 2000 a 2010. Utilizaram-se as fichas clínico-cirúrgicas dos cães e registraram-se os dados referentes a cada animal. Foram estudadas 342 articulações nos registros de 210 animais. A luxação medial congênita bilateral foi a apresentação mais frequente, e as fêmeas foram mais acometidas. A idade dos animais variou de 32 dias a 16 anos, com maior ocorrência da luxação nos animais com até um ano de idade e massa corporal inferior a 9,1kg. Em 11,7\% dos membros, a luxação era de grau I, em 39,8\% grau II, em $20,5 \%$ grau III e em $28 \%$ grau IV. A frequência de claudicação pré-operatória foi maior nos cães com luxações graus III e IV. Das 342 articulações, 218 foram submetidas à cirurgia. Na avaliação pós-operatória, os animais com luxação grau IV mostraram maior frequência de claudicação. A luxação patelar acomete principalmente fêmeas, sendo a luxação congênita bilateral a apresentação mais comum, e animais jovens e de pequeno porte são mais susceptíveis. A recuperação funcional pós-operatória nas luxações grau IV é lenta e parcial.
\end{abstract}

Palavras-chave: luxação, patela, cães, epidemiologia, ortopedia

\begin{abstract}
The epidemiological profile, clinical signs, and surgical techniques used and the recovery of dogs presented for treatment of patellar luxation in the Veterinary Hospital of UFMG in the period from 2000 to 2010 were evaluated through a retrospective study. The clinical and surgical records of dogs and the data recorded for each animal were used. 342 joints in the records of 210 animals were studied. The congenital bilateral medial luxation was the most frequent occurrence and females were more affected. The age of the animals ranged from 32 days to 16 years, with higher incidence of luxation in animals under one year of age and body mass below $9.1 \mathrm{~kg}$. In $11.7 \%$ of the members had grade I luxation, 39.8\% were grade II, $20.5 \%$ were grade III and $28 \%$ were grade IV. The frequency of preoperative claudicating was higher in dogs with luxation grades III and IV. Of the 342 joints, 218 underwent surgery. In the postoperative evaluation of animals, those with grade IV luxation showed higher claudicating frequency. The patellar luxation mainly affects females, and the bilateral congenital luxation is the most common occurrence, and young and small animals are more likely to have it. Functional recovery postoperative luxation in grade IV is slow and partial.
\end{abstract}

Keywords: luxation, patella, dogs, epidemiology, orthopedics

Recebido em 10 de abril de 2012

Aceito em 7 de maio de 2013

E-mail: jularavet@gmail.com 


\section{INTRODUÇÃO}

A luxação de patela ocorre frequentemente em cães e ocasionalmente em gatos, e é um evento comum na prática diária da traumatologia e ortopedia veterinária (Roush, 1993). Essa afecção pode ser classificada em lateral ou medial e em congênita ou traumática (Hulse, 1981; Denny e Butterworth, 2000; Lafond et al., 2002; Piermattei et al., 2006). A forma medial congênita é a mais comum em pequenos animais (Hulse, 1981; Piermattei et al., 2006) e ocorre principalmente em cães das raças toy e miniatura (Anderson, 1994; Denny e Butterworth, 2000). Em animais de grande porte ou gigantes, a luxação lateral ocorre com maior frequência, embora se relate aumento de casos de luxação medial nestas raças. De acordo com as alterações presentes, a luxação patelar pode ser classificada em quatro graus (Putnam, 1968; Singleton, 1969). Essa classificação auxilia no diagnóstico e na seleção do tratamento cirúrgico. As técnicas cirúrgicas utilizadas para tratamento da luxação patelar dependem do grau de luxação e das anormalidades presentes, sendo o tratamento conservativo geralmente limitado aos cães com luxação grau I sem sinais de claudicação (Hulse, 1981). A avaliação dos resultados cirúrgicos é baseada na melhora clínica, na qualidade da locomoção e no aspecto radiográfico (Roush, 1993). O estudo clínicocirúrgico e epidemiológico da doença abrangendo um número elevado de animais já foi descrito em outros países, porém ainda faltam estudos similares para caracterizá-la no Brasil. Tendo em vista a alta frequência da luxação patelar, considerada uma das patologias ortopédicas mais comuns em cães, o objetivo deste trabalho foi avaliar, por meio de um estudo retrospectivo, o perfil epidemiológico, os sinais clínicos, bem como o tratamento cirúrgico utilizado e a recuperação pós-operatória em cães apresentados para tratamento no Hospital Veterinário da UFMG, no período de 2000 a 2010.

\section{MATERIAL E MÉTODOS}

Foi realizado um estudo retrospectivo, baseado no levantamento de fichas clínico-cirúrgicas de cães apresentados para tratamento de luxação de patela, no período compreendido entre janeiro de 2000 e julho de 2010, no Hospital Veterinário da UFMG. Foram registrados os dados referentes a sexo, raça, idade e peso no momento do atendimento, histórico com observações referentes aos sinais clínicos apresentados e à duração deles, grau e tipo da luxação patelar (medial ou lateral), membro acometido (direito e/ou esquerdo), etiologia da luxação (congênita ou traumática), tratamento realizado e tempo médio de recuperação dos animais após a cirurgia. Informações referentes ao pósoperatório dos animais foram separadas em dois períodos: até 60 dias de pós-operatório e de 60 a 180 dias. A qualidade da locomoção no pósoperatório foi dividida em escores de claudicação adaptados de Gibbons et al. (2006), como apresentado na Tab. 1. Os animais foram divididos em três categorias de peso, segundo Gibbons et al. (2006): massa corporal igual ou inferior a $9,1 \mathrm{~kg}$, considerados cães de pequeno porte; entre 9,1 e $18,2 \mathrm{~kg}$, considerados de médio porte; e acima de $18,2 \mathrm{~kg}$, considerados cães de grande porte.

Tabela 1. Sistema de pontuação para as características de claudicação e apoio do membro após cirurgia de luxação de patela em cães atendidos no Hospital Veterinário da UFMG, no período de 2000 a 2010

\begin{tabular}{|c|c|}
\hline \multicolumn{2}{|r|}{ Claudicação e apoio do membro } \\
\hline Pontuação & Descrição \\
\hline 1 & Apoio normal do membro em estação. Ausência de claudicação ao caminhar e correr \\
\hline 2 & $\begin{array}{l}\text { Claudicação discreta ou intermitente com alívio discreto do suporte de peso no membro e } \\
\text { mínima rotação da tuberosidade tibial até } 30^{\circ}\end{array}$ \\
\hline
\end{tabular}

Claudicação moderada com alívio do suporte de peso no membro e rotação da tuberosidade tibial de 30 a $60^{\circ}$ dígitos ou ausência de apoio. Rotação da tuberosidade tibial de 60 a $90^{\circ}$ 
As diferenças de frequências entre tipo de luxação (medial ou lateral), acometimento uni ou bilateral, grau da luxação, massa corporal do animal, idade, presença de claudicação e idade de diagnóstico da luxação foram analisadas pelo teste do qui-quadrado. A significância foi estabelecida em $\mathrm{P}<0,05$. As demais observações, como raça, sinais clínicos, membro acometido, as técnicas cirúrgicas utilizadas e a recuperação do animal no pós-operatório foram avaliadas pela análise descritiva.

\section{RESULTADOS E DISCUSSÃO}

Encontrou-se o registro de luxação de patela em 210 animais, correspondendo a 342 articulações. A luxação medial ocorreu em $302(88,3 \%)$ das 342 articulações e em $184(87,6 \%)$ dos 210 animais avaliados. A luxação medial foi, como já relatado na literatura (DeAngelis e Hohn, 1970; Remedios et al., 1992; Hayes et al.,1994; 2001; Gareth e Sorrel, 2006; Gibbons et al., 2006; Piermattei et al., 2006; Mostafa et al., 2008), a de maior ocorrência também neste estudo, com diferença significativa $(\mathrm{P}<0,05)$. Nenhuma associação foi encontrada entre o tipo de luxação (medial ou lateral) e o grau da luxação $(\mathrm{P}>0,05)$. Segundo Olmstead (1993) e Piermattei et al. (2006), a luxação medial congênita representa 75 a $80 \%$ das luxações patelares em cães, sendo o tipo mais comum independentemente da raça, apesar de ser observada predominantemente em cães de pequeno porte (Denny, 1993; Anderson, 1994). Entretanto, relata-se um aumento significativo da luxação medial em raças de grande porte e gigantes, especialmente em Akita, Labrador e Husky (Piermattei et al., 2006). No presente estudo, 14 animais $(6,6 \%)$ de grande porte, entre eles três Pit Bull, três Labradores, dois Rottweiler, dois Mastim Napolitano, dois Filas Brasileiros, um Akita e um Dog de Bordeaux, apresentaram luxação medial de patela.

Dos animais com luxação medial, as raças mais acometidas foram: Poodle (74 cães) $(40,2 \%)$, Pinscher (29 cães) $(15,8 \%)$ e Yorkshire (23 cães) $(12,5 \%)$, resultados similares aos apresentados na literatura (Priester, 1972; Hayes et al., 1994; Souza et al., 2010).

A luxação lateral ocorreu em $40(11,7 \%)$ das 342 articulações e em $26(12,4 \%)$ dos 210 cães avaliados, índice superior ao relatado por
Remedios et al. (1992). As raças mais acometidas foram Poodle (12 cães) (46\%) e Yorkshire (quatro cães) (15,4\%). A luxação lateral é citada principalmente em animais de raças de grande porte e gigantes (Hulse, 1981; Slocum e Slocum, 1998), e de menor ocorrência em relação à luxação medial (DeAngelis e Hohn, 1970; Hulse, 1981; Vasseur, 2003; Piermattei et al., 2006). Na presente pesquisa, a luxação lateral ocorreu predominantemente em cães de pequeno porte, com massa corporal igual ou inferior a $9,1 \mathrm{~kg}(78,3 \%$ dos casos $)$, contrastando com a citação de Padilha Filho et al. (2005) e Vasseur (2003), que relatam tratar-se de um caso raro de luxação e de maior ocorrência em cães de grande porte.

Todas as luxações observadas neste estudo eram de origem congênita, a exemplo dos relatos de Gibbons et al. (2006) e Gareth e Sorrel (2006). Embora a luxação não esteja presente ao nascimento, as anormalidades anatômicas que a causam estão (Piermattei et al., 2006). As anormalidades anatômicas observadas nos animais do presente estudo foram deslocamento medial ou lateral do quadríceps, rotação e encurvamento do fêmur e da tíbia, contratura de quadríceps e rotação interna ou externa das patas, como citam outros autores (Vasseur, 2003; Padilha Filho et al., 2005 e Piermattei et al., 2006).

Cinquenta animais (24\%) apresentaram luxação unilateral, enquanto 160 (76\%) luxação bilateral, o que leva à constatação de que a luxação bilateral é significativamente mais frequente $(\mathrm{P}<0.05)$. Além disso, o acometimento bilateral aumentou significativamente $(\mathrm{P}<0,05)$ com a gravidade da luxação, ou seja, nas luxações de graus III e IV, a probabilidade de acometimento em ambos os membros é maior. O membro posterior esquerdo foi acometido em $51 \%$, e o direito em $49 \%$ dos casos, semelhante ao encontrado por Gibbons et al. (2006) e Gareth e Sorrel (2006).

Com referência ao sexo, 126 cães $(60,0 \%)$ eram fêmeas, e $84(40,0 \%)$ machos. A razão entre fêmeas e machos foi de 1.5:1 para todos os cães avaliados, 1.5:1 para cães com menos de $15 \mathrm{~kg}$ e 1.4:1 para cães com massa corporal superior a $15 \mathrm{~kg}$. A razão entre fêmeas e machos não foi influenciada pela massa corporal do animal e, portanto, não corrobora a citação de que a 
luxação patelar seja mais comum em fêmeas de pequeno porte e em machos de grande porte, como relatado por Gibbons et al. (2006) e Souza et al. (2010), mas está de acordo com a citação de Roush (1993) de que as fêmeas são mais acometidas em relação aos machos. Esta maior frequência em fêmeas pode estar relacionada à influencia hormonal ou, alternativamente, ser uma expressão associada ao cromossomo $\mathrm{X}$ (Priester, 1972).

A idade dos animais variou de 32 dias a 16 anos. Verificou-se diferença entre as faixas etárias estudadas, com aumento significativo da luxação de patela nos animais jovens com até um ano de idade $(\mathrm{P}<0,05)$. Por se tratar de uma afecção caracterizada como congênita, cujas anormalidades anatômicas já estão presentes ao nascimento (Piermattei et al., 2006), justifica-se a manifestação da luxação de patela predominantemente em animais jovens. Ao se separarem dois grupos de animais de acordo com a faixa etária, observou-se que os animais com luxação grau IV são estatisticamente mais jovens, com idade máxima de 24 meses, em relação aos animais com luxação de graus I, II e III, como relatado por Souza et al. (2010).

A massa corporal dos animais variou de $400 \mathrm{~g}$ a $56 \mathrm{~kg}$, com média de $7,3 \mathrm{~kg}$. Este valor é semelhante ao encontrado por Souza et al. (2010), mas inferior aos apresentados por Heather et al. (2005), Gareth e Sorrel (2006) e Mostafa et al. (2008), que apresentaram médias de $14,1,12,3$ e $9,8 \mathrm{~kg}$, respectivamente. No presente estudo, 80,8\% dos cães $(n=168)$ com luxação de patela são de pequeno porte (massa corporal até $9,1 \mathrm{~kg}), 7,7 \% \quad(\mathrm{n}=16)$ entre 9,1$18,2 \mathrm{~kg}$, considerados de médio porte, e $11,5 \%$ $(n=24)$ de grande porte, com massa corporal acima de $18,2 \mathrm{~kg}$, porém nenhuma relação significativa foi observada entre a massa corporal do animal e o grau de luxação $(\mathrm{P}>0,05)$.

Diferentemente do relatado por Hayes et al. (1994), neste levantamento não houve diferença entre cães com massa corporal igual ou inferior a $20 \mathrm{~kg}$ ou superior a $20 \mathrm{~kg}$ em relação à idade de diagnóstico da luxação, ao se considerarem animais de até dois anos e acima de dois anos de idade $(\mathrm{P}>0,05)$. Segundo os autores, os cães de grande porte são diagnosticados mais precocemente em relação aos de médio ou pequeno porte. Tal observação pode estar relacionada à maior percepção das deformidades anatômicas e manifestação precoce dos sinais clínicos nos cães de grande porte que, geralmente, apresentam crescimento rápido, ou pode estar associada ao maior peso e, consequentemente, à claudicação mais evidente.

Quanto ao grau de luxação, 40 membros $(11,7 \%)$ mostraram luxação de grau I, $136(39,8 \%)$ de grau II, 70 (20,5\%) de grau 3 e 96 (28\%) luxação de grau IV. A luxação de grau II foi a mais comumente observada, como citam outros autores (Gareth e Sorrel, 2006; Gibbons et al., 2006; Mostafa et al., 2008; Souza et al., 2010), seguida da luxação de grau IV. É comum as luxações de grau I serem encontradas ocasionalmente durante o exame clínico em animais de pequeno porte (Roush, 1993; Vasseur, 2003; Piermattei et al., 2006) e associadas com sinais clínicos discretos, que podem passar despercebidos pelo proprietário (Roush, 1993). A luxação de patela grau II cursa geralmente com claudicação intermitente (Roush, 1993; Vasseur, 2003), e alterações anatômicas podem estar presentes, sendo, portanto, neste estágio que grande parte dos proprietários observa ou dá maior importância à claudicação e procura um tratamento.

Os sinais clínicos mais citados pelos proprietários foram claudicação intermitente ou constante, ausência de apoio, ou seja, os animais carregam os posteriores com apoio exclusivo nos anteriores, dificuldades em subir e descer degraus, rotação e flexão dos membros posteriores, posterior rebaixado, dorso curvado, dor à palpação e redução da massa muscular, semelhante ao observado por Padilha filho et al. (2005). A frequência de claudicação préoperatória aumentou com a gravidade da luxação e foi significativamente maior nos graus III e IV $(\mathrm{P}<0,05)$.

Das 342 articulações diagnosticadas com luxação de patela, o procedimento cirúrgico foi realizado em 218 com luxação medial e em 34 com luxação lateral. As demais 90 articulações não foram submetidas ao procedimento cirúrgico por motivos não especificados.

As técnicas cirúrgicas empregadas foram reforço do ligamento femoropatelar com retalho de fáscia lata autóloga, imbricação da cápsula, sutura antirrotatória fabelotibial, desmotomia, 
aprofundamento da tróclea, transposição da tuberosidade da tíbia e osteotomias corretivas do fêmur ou da tíbia. Combinações de duas ou mais dessas técnicas cirúrgicas mencionados foram utilizadas em 98,8\% dos casos para correção da luxação, como indicado pelos autores Padilha Filho et al. (2005), Gibbons et al.(2006), Gareth e Sorrel (2006), Piermattei et al. (2006) e Souza et al. (2010).

Em 13 animais com luxação grau I $(81,25 \%)$ e em três casos de luxação grau II $(2,75 \%)$ na presente pesquisa, a técnica utilizada foi o uso do retalho de fáscia lata autóloga para reforço do ligamento femoropatelar associado à imbricação da cápsula. Em um animal $(33,3 \%)$ com luxação grau II, ocorreu a reincidência da luxação, sugerindo que neste grau deve-se considerar a associação da transposição da tuberosidade da tíbia ou a sutura antirrotatória para melhor estabilização articular. Em três articulações com luxação grau I $(18,75 \%)$, a patela manteve-se estável no sulco troclear apenas com o emprego da imbricação da cápsula articular. Segundo Roush (1993), a imbricação da cápsula é realizada para correção de luxação em todos os graus e pode ser o único procedimento necessário para as luxações de grau I ou II.

Em todos os casos de luxação grau II foi usada a imbricação da cápsula associada ao reforço do ligamento femorotibiopatelar com fáscia lata, sendo a técnica empregada como único tratamento em três $(2,75 \%)$ casos. Nos demais membros $(97,25 \%)$, foram feitas diferentes associações segundo a necessidade do caso, como a desmotomia $(97,3 \%)$, o aprofundamento da tróclea $(55 \%)$, a transposição da tuberosidade tibial $(50 \%)$ e a sutura antirrotatória fabelotibial (34\%). Em todos os casos de luxação graus III e IV, as técnicas utilizadas foram o reforço do ligamento femoropatelar, a imbricação da cápsula, a desmotomia, o aprofundamento do sulco troclear e a transposição da tuberosidade da tíbia. A sutura antirrotatória foi empregada em $24 \%$ dos casos de luxação grau III e em $46 \%$ dos de luxação grau IV, quando a transposição da tuberosidade da tíbia, em sua máxima extensão sugerida $(2-4 \mathrm{~mm})$, não propiciava um alinhamento satisfatório do membro.

A desmotomia é necessária em todos os graus III e IV e em muitos casos de grau II (Tomlinson e Constantinescu, 1994), uma vez que ela alivia a contratura da cápsula articular (Hulse, 1981; Roush, 1993; Piermattei et al., 2006). A correção das deformidades ósseas é considerada fundamental para o sucesso do tratamento da luxação patelar (Read, 1999), como observado também neste estudo. Entre os procedimentos de reconstrução óssea são mais comumente referidos o aprofundamento do sulco troclear, a transposição da tuberosidade tibial e as osteotomias corretivas do fêmur e da tíbia (Roush, 1993; Piermattei et al., 2006).

A técnica de aprofundamento da tróclea no presente estudo foi realizada em todos os animais com luxação graus III e IV e na maioria dos de grau II. A pressão insuficiente ou ausente deste sesamoide sobre a tróclea femoral ocasiona o rasamento do sulco, e a luxação intermitente provoca a diminuição da borda troclear medial ou lateral, segundo a direção da luxação, e desgaste da cartilagem do côndilo femoral. A deformidade troclear é corrigida, sendo que a profundidade do novo sulco deve acomodar pelo menos $50 \%$ da patela. O desafio da correção dessa deformidade é quando há perda da convexidade da patela, que, consequentemente, não produzirá efeito sobre a trocleoplastia, pois não há superfície patelar para encaixe. Nesses casos, a prótese patelar seria a solução mais recomendada, pois manteria a biomecânica do mecanismo extensor do quadríceps.

No presente estudo, a transposição da tuberosidade da tíbia foi feita em todos os casos de luxação graus III e IV e na metade dos casos de luxação grau II, com o objetivo de estabelecer o alinhamento entre o grupo muscular quadríceps, o sulco troclear e a tuberosidade tibial. Essa transposição é requerida quando ela está posicionada lateral ou medialmente em relação à origem de inserção do grupo muscular quadríceps (Roush, 1993).

Entre os animais com luxação grau IV, 16 (6,3\%) foram submetidos à osteotomia do fêmur, e dois $(0,8 \%)$ à osteotomia da tíbia. As osteotomias foram efetuadas, em geral, em forma de cunha e estabilizadas com o emprego de placas, parafusos ou pinos de Rush. Somente três animais eram adultos, e os demais apresentavam idade inferior a um ano. Nos casos de curvatura acentuada do fêmur distal e/ou da tíbia proximal, é necessário associar-se a osteotomia do fêmur ou da tíbia para restaurar o alinhamento normal 
do mecanismo extensor em pacientes com luxação grave (Denny e Butterworth, 2000; Piermattei et al., 2006), como observado nesta pesquisa e também relatado por Roch e Gemmil (2008) e Souza et al. (2010). O não alinhamento do eixo pode levar à reincidência da luxação patelar.

Duzentas e onze articulações tratadas cirurgicamente foram examinadas clinicamente quanto à claudicação e ao apoio do membro entre uma e oito semanas de pós-operatório. Oitenta e um por cento dos animais com luxação grau I apresentaram pontuação 2 no escore de claudicação, enquanto $57,3 \%$ e $41 \%$ dos animais com luxação graus II e III, respectivamente, possuíam ausência de claudicação ao caminharem ou correrem (pontuação 1). A claudicação neste período foi mais acentuada em $30,4 \%$ dos animais com luxação grau IV, que apresentaram pontuação 4 na avaliação, como demonstrado na Tab. 1.

Tabela 2. Frequência da claudicação de acordo com o sistema de pontuação entre uma e oito semanas de pós-operatório em relação ao grau de luxação em cães com luxação patelar atendidos no Hospital Veterinário, no período de 2000 a 2010

\begin{tabular}{ccccc}
\hline \multirow{2}{*}{$\begin{array}{c}\text { Sistema de } \\
\text { pontuação }\end{array}$} & \multicolumn{4}{c}{ Grau de luxação (\%) } \\
\cline { 2 - 5 } & I & II & III & IV \\
\hline 1 & $8(16,7 \%)$ & $39(57,4 \%)$ & $16(41,0 \%)$ & $13(23,2 \%)$ \\
3 & $39(81,2 \%)$ & $12(17,6 \%)$ & $8(20,5 \%)$ & $14(25,0 \%)$ \\
4 & 0 & $12(17,6 \%)$ & $8(20,5 \%)$ & $12(21,4 \%)$ \\
& $1(2,1 \%)$ & $5(7,40 \%)$ & $7(18,0 \%)$ & $17(30,4 \%)$ \\
\hline
\end{tabular}

Oitenta e três articulações tratadas cirurgicamente foram reexaminadas após os 60 dias de pós-operatório. Para todos os graus avaliados, a maioria dos animais apresentou melhor desempenho em relação à qualidade de locomoção e foi enquadrada na pontuação $1 \mathrm{da}$ tabela. Entretanto, foi observada uma alta frequência de animais com luxação grau IV $(29,2 \%)$, com acentuada transferência do suporte do peso para os membros anteriores e pontuação 4 no escore de claudicação e apoio do membro no período avaliado (Tab. 2). Em geral, o sucesso do tratamento é inversamente proporcional ao grau de luxação (Remedios et al., 1992; Roush, 1993; Gareth e Sorrel, 2006). Luxações de graus I, II ou III possuem, na maioria dos casos, um prognóstico favorável (Willauer e Vasseur, 1987), enquanto as luxações de grau IV apresentam um prognóstico reservado a desfavorável (DeAngelis e Hohn, 1970), em virtude da extensão das anormalidades presentes (Tomlinson e Constantinescu, 1994).

Tabela 3. Frequência da claudicação de acordo com o sistema de pontuação após 60 dias de pósoperatório em relação ao grau de luxação em cães com luxação patelar atendidos no Hospital Veterinário, no período de 2000 a 2010

\begin{tabular}{ccccc}
\hline \multirow{2}{*}{$\begin{array}{c}\text { Sistema de } \\
\text { pontuação }\end{array}$} & \multicolumn{4}{c}{ Grau de luxação $(\%)$} \\
\cline { 2 - 4 } & I & II & III & IV \\
\hline 1 & $4(66,6 \%)$ & $23(74,2 \%)$ & $12(54,6 \%)$ & $11(45,8 \%)$ \\
2 & 0 & $3(9,70 \%)$ & $5(22,7 \%)$ & $5(20,8 \%)$ \\
3 & $1(16,7 \%)$ & $3(9,70 \%)$ & $1(4,50 \%)$ & $1(4,20 \%)$ \\
4 & $1(16,7 \%)$ & $2(6,40 \%)$ & $4(18,2 \%)$ & $7(29,2 \%)$ \\
\hline
\end{tabular}

\section{CONCLUSÕES}

Com base na análise dos dados fornecidos por este estudo, conclui-se que a luxação patelar acomete principalmente fêmeas, sendo a luxação congênita medial bilateral a apresentação mais comum, e animais jovens e de pequeno porte são mais susceptíveis a essa patologia. A recuperação funcional pós-operatória do membro nas luxações de grau IV é lenta e parcial. 


\section{AGRADECIMENTOS}

À Escola de Veterinária da UFMG, por todo o acervo disponível para realização deste trabalho, e ao Conselho Nacional de Desenvolvimento Científico e Tecnológico $(\mathrm{CNPq})$, pelo apoio à pesquisa.

\section{REFERÊNCIAS}

ANDERSON, J. The stifle. In: HOULTON, J.E.F.; COLLINSON, R.W. Manual of Small Animal Veterinary Assocation. Gloucestershire: British Small Animal Veterinary Association, 1994. p.275-279.

DeANGELIS, M.; HOHN, R.B. Evaluation of surgical correction of canine patellar luxation in 142 cases. $J$. Am. Vet. Med. Assoc., v.156, p.587-594, 1970.

DENNY, H.R. Aguide to canine and feline orthopaedic surgery. 3.ed. Oxford: Blackwell Scientific, 1993. p.337-341.

DENNY, H.R.; BUTTERWORTH, S.J. The Stifle. In: . A guide to canine and feline orthopaedic surgery. 4.ed. Oxford:Blackwell Science, 2000. p.512553.

GARETH, I.A.; SORREL, J.L.H. Complications Associated with Corrective Surgery for Patellar Luxation in 109 Dogs. Vet. Surg., v.35, p.559-566, 2006.

GIBBONS, S.E.; MACIAS, C.; TONZING, M.A. et al. Patellar luxations in 70 large breed dogs. J. Small Anim. Pract., v.47,p.3-9, 2006.

HAYES, A.G.; BOUDRIEAU, R.J.; HUNGERFORD, L.L. Frequency and distribution of medial and lateral patellar luxation in dogs: 124 cases (1982-1992). $J$. Am. Vet. Med. Assoc., v.57, p.105-109, 1994.

HEATHER, A.T.; GRIFFON, D.J.; THOMAS, M.W. et al. Pre and Postoperative Radiographic and Computed Tomographic Evaluation of Dogs with Medial Patellar Luxation. Vet. Surg., v.34, p.265-272, 2005.

HULSE, D.A. Pathophysiology and management of Medial Patelar Luxation in the dog. Vet. Med. Small Anim. Clin., v.76, p.43-51, 1981.

LAFOND, E.; BREUR, G.J.; AUSTIN, C.A. Brees susceptibility for developmental orthopaedic diseases in dogs. J. Am. Anim. Hosp. Assoc., v.38, p.467-477, 2002.

MOSTAFA, A.A.; GRIFFON, D.J.; THOMAS, M.W. et al. Proximodistal Alignment of the Canine Patella: Radiographic Evaluation and Association with Medial and Lateral Patellar Luxation. Vet. Surg., v.31, p.201$211,2008$.
OLMSTEAD, M.L: Lateral luxation of the patella. In: BOJRAB, M. Disease Mechanisms in Small Animal Surgery. 2.ed . Philadelphia: Lea \& Febiger, 1993. p.818-820.

PADILHA FILHO, J.G.; DÓREA NETO, F.A.; DÓREA, H.C. et al. Treatment of the lateral patellar luxation in toy poodles. Cienc. Rural, v.35, p.843-847, 2005 .

PIERMATTEI, D.L.; FLO, G.L.; DECAMP. C.E. The Stifle Joint. In: _. Handbook of Small Animal Orthopedics and Fracture Repair. 4.ed. Philadelphia: Saunders, 2006. p.562-632.

PRIESTER, W.A. Sex, size, and breed as risk factors in canine patellar dislocation. $J$ Am Vet Med Assoc., v.160, p.740-742, 1972.

PUTNAM, R.W. Patellar luxation in the dog. 1968. 111f. Dissertação (Mestrado em Medicina Veterinária) - University of Guelph, Guelph.

READ, R.A. Opciones racionales de tratamiento de la luxación interna de rótula. Waltham Focus, v.9, p.2531, 1999.

REMEDIOS, A.M.; BASHER, A.W.P.; RUNYON, C.L. et al. Medial patellar luxation in 16 large dogs: A retrospective study. Vet. Surg., v.21, p.5-9, 1992

ROCH, S.P.; GEMMILL, T.J. Treatment of medial patellar luxation by femoral closing wedge ostectomy using a distal femoral plate in four dogs. J. Small An. Pract., v.49, p.152-158, 2008.

ROUSH, J.K. Canine patellar luxation. Vet. Clin. N. Am. Small Anim. Pract., v.23, p.855-868, 1993.

SINGLETON, W.B. The surgical correction of stifle deformities in the dog. J. Small Anim. Pract., v.10, p.59-69, 1969.

SLOCUM, B.; SLOCUM, T.D. Knee. In: BOJRAB, M.J. Current techniques in small animal surgery. 4.ed. Philadelphia: Lippincott Williams \& Wilkins, 1998. p.1187-1244.

SOUZA, M.M.D.; RAHAL, S.C.; PADOVANI, C.R. et al. Estudo retrospectivo de cães com luxação patelar medial tratados cirurgicamente. Cienc. Rural, v.40, p.31-36, 2010.

TOMLINSON, J.; CONSTATINESCU, G.M. Repair of medial patellar luxation. Vet. Med., v.89, p.48-56, 1994.

VASSEUR, P.B. Stifle Joint. In: SLATER, D. Textbook of Small Animal Surgery. 3. ed. Philadelphia: Saunders, 2003. p.2090-2133.

WILLAUER, C.C.; VASSEUR, P.B. Clinical Results of Surgical Correction of Media Luxation of the Patella in Dogs. Vet. Surg., v.16, p.31-36, 1987. 